\title{
Caractéristiques épidemio- clinique et diagnostique des patients décédés au service de pneumo-phtisiologie du centre hospitalier universitaire du point $\mathrm{g}$.
}

\section{Epidemiological, clinical and diagnostic characteristics of patients died in pneumophtisiology department at point $\mathrm{g}$ university hospital.}

\author{
Sissoko BF ${ }^{1}$, Baya $B^{1}$, Soumaré $D^{1}$, Ouattara $K^{1}$,Kanouté $T^{1}$, M'Baye $O^{1}$, Dao $E^{1}$, Toloba $Y^{1}, D^{\prime}$ iallo $S^{1}$. \\ 1. Service de Pneumo-phtisiologie du Centre Hospitalier Universitaire (CHU) du Point G, \\ Correspondant: Docteur Bocar Baya, Médecin , Centre Hospitalier Universitaire du Point G, Boite Postale = \\ 333 Bamako, Mali. \\ Adresse email : bbaya@icermali.org Téléphone portable : (00223) 66747270
}

\section{RESUME:}

Objectif: Naus avons conduit une étude rétrospective, descriptive pour étudier les caractéristiques épidémiologiques, cliniques et paracliniques des patients décédés dans le service.

Patients et méthode : Nous avons colligé les dossiers des patients décédés au cours de leur hospitalisation dans le service de pneumo-phtisiologie du centre hospitalo-universitaire du point G entre juin 2010 et juin 2012. Résultats : Sur un total de 1025 patients hospitalisés pendant la période d'étude, 307 sont décédés soit une fréquence de 29,95\%. Seuls 189 dossiers répondaient à nos critères soit 18,44\%. La mortalité globale était de $29,95 \%$, avec une prédominance du sexe masculin (sex-ratio $=3,39$ ). La tranche d'âge la plus élevée était de 60 à 90 ans $(32,3 \%)$ des cas. Une radiographie thoracique de face pathologique dans $98,4 \%$ des cas avec des opacités occupant au moins les $2 / 3$ du champ pulmonaire dans $39,8 \%$ et bilatérales dans $66,7 \%$ des cas. La durée moyenne de séjour dans le service était de 18,6 jours. La tuberculose était le diagnostic de deces le fréquemment retrouvée avec $28 \%$ des cas.

Conclusion : Malgré les efforts fournis dans le cadre des objectifs du millénaire, notre pays continu à payer un lourd tribut à la Tuberculose.

Mot clés : Décès à l'hôpital, épidémiologie, clinique, paraclinique.

\section{SUMMARY:}

Objective: We conducted a retrospective, descriptive to study the epidemiological, clinical and paraclinical characteristics of patients who died in the department. Patients and method: We collected the records of patients who died during their hospitalization in Pneumophtisiology department at Point G Teaching Hospital between June 2010 and June 2012.

Results: Of a total of 1025 patients hospitalized during the study period, 307 died with a frequency of $29.95 \%$. Only 189 cases met our criteria $(18.44 \%$ ). The overall mortality was $29.95 \%$, with a predominance of males (sex ratio $=3.39$ ). The highest age range was 60 to 90 years $(32.3 \%)$. Pathological face chest radiography was found in $98.4 \%$, opacities occupying at least $2 / 3$ of the lung in $39.8 \%$ and bilateral in $66.7 \%$ of cases. The average length of stay in department was 18.6 days. Pulmonary tuberculosis was the most cause of death with $28 \%$ of cases.

Conclusion: Despite the efforts made in the context of the Millennium Goals, our country continues to pay a heavy price for tuberculosis.

Key words: hospital deaths, epidemiology, clinical, paraclinical.

\section{INTRODUCTION}

Les affections touchant ces différentes parties du poumon sont différentes de par leur étiologie, les plus impliqués étant d'origine infectieuse, tumorale et ou d'évolution chronique [1].Dans les pays développés les maladies respiratoires chroniques occupent une place importante, la prévalence des broncho-pneumopathies chroniques obstructives (BPCO) dans le monde est estimée à 9,33/1000 chez les hommes et 7,39/1000 chez les femmes; celle de l'asthme varie de 1 à 9,7/1000 selon les régions [2]. Sur les 4 millions de décès annuels par maladie respiratoire aigüe enregistrée dans le monde, $98 \%$ sont imputables aux infections respiratoires basses [3]. Les maladies respiratoires les plus fréquentes sont l'asthme, les cancers broncho-pulmonaires et la BPCO, leur principal facteur de risque est le tabagisme [4].En France, les maladies respiratoires sont responsables chaque année de 37000 décès, soit $6 \%$ de l'ensemble des décès $(10 \%$ si l'on inclut les tumeurs de l'appareil respiratoire) et sont à l'origine de $7 \%$ de séjours hospitaliers et 7 séances sur 100 en médecine libérale [5]. Les infections respiratoires de l'adulte ont une place importante en termes de morbidité et d'arrêt de travail. Elles occupent le $2^{\text {ème }}$ rang des pathologies déclarées après les atteintes dentaires [6].Les pneumonies ont un taux d'incidence qui varie de 100 à 500 pour 100000 adultes. La 
mortalité reste élevée, de l'ordre de 6 à $20 \%$ selon les études [7]. En France, comme dans tous les pays industrialisés, l'incidence des infections à mycobactéries tend à se stabiliser. Elle est plus élevée dans les populations émigrées et les sujets de plus de 65 ans [8]. En Afrique, la prévalence des BPCO chez les malades hospitalisés dans des services spécialisées allait de $2,7 \%$ en Guinée Conakry à $14 \%$ au Maroc [9]. Dans ces pays, les pathologies respiratoires infectieuses dont la tuberculose demeure un problème de santé publique [10]. Une étude réalisée en Côte d'ivoire de 1998 à 2007 a objectivé un taux élevé de décès en hospitalisation pneumologique de $21,15 \%$ avec une mortalité plus importante liées aux pathologies associant une forte incidence de la coïnfection par le VIH [11]. Au Mali en 2001 une étude a trouvé $13,85 \%$ de décès liés aux maladies respiratoires [12] et en 2006, une autre étude montre que 4,76\% des décès étaient dus aux pathologies de l'appareil respiratoire [13]. Les affections respiratoires chroniques ont un taux de mortalité de 85,9 pour 100000 habitants [14] et les pneumopathies non tuberculeuses en progression représentaient la $2^{\text {ème }}$ cause de morbi-mortalité en 2003 [15]. Au service de pneumologie du CHU du Point G, la tuberculose demeure la principale cause d'hospitalisation [16]. Devant le fait qu'aucune étude similaire n'ait été réalisée auparavant dans le service, nous nous sommes proposé de faire une revue des dossiers des malades décédés au cours de leur hospitalisation avec pour objectif d'identifier les différentes caractéristiques socio démographiques, cliniques et para cliniques des patients décédés dans le service.

\section{MATERIEL ET METHODE}

Il s'agissait d'une étude rétrospective et descriptive, allant de Juin 2010 à juin 2012 dans le service de pneumo-phtisiologie du centre hospitalouniversitaire (CHU) du Point G. Le service de pneumologie est compose d'une unité de tuberculose ou sont hospitalises les cas confirmés et les cas en traitement ambulatoire dont l'état général est altéré quelque soit la raison (effets secondaires ou autres situations). Les médicaments antituberculeux et les frais d'hospitalisation sont gratuits pour le malade. Une unité de pathologies pulmonaires non tuberculeuses et les cas suspects tuberculeux non confirmés. Nous avons colligé tous les dossiers des patients de tout âge et de sexe confondus ayant été hospitalisés dont le diagnostic a été confirmé dans le service pendant notre période d'étude et l'évolution marquée par le décès du patient.L'échantillonnage a été exhaustif et a concerné les dossiers des patients hospitalisés et décédés pendant leur séjour dans le service.

Critères d'inclusion:

Etaient inclus dans notre étude les dossiers des patients hospitalisés depuis au moins soixantedouze heures décédés des deux sexes, âgés de quinze ans au moins, ayant un dossier complet comportant les caractéristiques socio démographiques du patient, les antécédents, un examen clinique complet et les résultats de certains examens para cliniques dont la radiographie pulmonaire de face, la numération formule sanguine (NFS) et de l'examen des crachats à la recherche de bacille acido-alcoolo-résistants (BAAR) et de la recherche des autres bactéries non tuberculeuse.

Critères de non inclusion: Etaient exclus de l'étude - les dossiers des patients décédés après un séjour de moins de 72 heures,

- les dossiers des patients décédés âgés de moins de 15 ans,

- les dossiers incomplets avec manque des données socio démographiques, cliniques et/ou de certains des examens para cliniques

Collecte et analyses : Les données ont été collectées à partir des dossiers médicaux des patients hospitalisés décédés dans le service pendant notre période d'étude et reportées sur des fiches d'enquête comportant plusieurs items. Les variables sociodémographiques (âge, sexe...etc.),

Variables cliniques (antécédents, diagnostic, durée d'hospitalisation....etc.)

Variables para cliniques (radiographie pulmonaire, NFS, crachat BAAR, etc.)

La saisie des données a été faite à partir du logiciel Epi Info version 3.5.3 et l'analyse statistique avec le logiciel SPSS version 11.0. En tenant compte de la distribution anormale du nombre des patients décédés selon la cause du décès, nous avons utilisé le test statistique de Krusskall Wallis pour comparer les médianes de la durée de séjour entre la date d'hospitalisation du patient dans le service et la date du décès en fonction des différentes causes de décès observées leur fréquence. Le seuil de significativité a été fixé à $5 \%$.

Considération éthiques : Les dossiers des patients étaient analysés dans le strict respect de leur confidentialité dans la salle des archives du service de pneumo-phtisiologie. Les bonnes pratiques médicales ont été respectées pendant la collecte des données (autorisation du chef de service pour l'accès aux dossiers, port de masque et des gants pour le tri des dossiers, reclassement des dossiers après triage).

\section{RESULTATS}

Au total 1025 patients ont été hospitalisés dans le service de pneumo-phtisiologie du centre hospitalouniversitaire du point $\mathrm{G}$; parmi eux, 307 malades sont décédés soit une fréquence de $29,95 \%$. Seuls 189 dossiers répondaient à nos critères soit $18,44 \%$. Les patients âgés de 60 à 95 ans étaient les plus représentés avec 32,3\%, l'âge moyen était de 50,7 ans(figure 2). La majorité des patients étaient des hommes à 77,2\% avec un sex-ratio à 3,39.avec des extrêmes de 17 et 90 ans(figure 3). Les principaux 
antécédents médicaux étaient la tuberculose pulmonaire à $12,2 \%$ et l'ulcère gastroduodénal à 11,6\%(Tableau I). Les pneumopathies bactériennes étaient le diagnostic d'hospitalisation le plus fréquent avec $28 \%$ suivies de la tuberculose pulmonaire avec 24,9\%(Tableau II). L'étendue des lésions radiologiques était supérieure au 2/3 des champs pulmonaires chez $39,8 \%$ des patients décédés (Tableau III). La tuberculose pulmonaire était le diagnostic de décès le plus fréquent avec $28 \%$ suivie des pneumopathies bactériennes avec 20,6\%(tableau IV). Les patients décédés pour infection tuberculeuse avaient un séjour plus long avec une médiane de 15 jours et ceux décédés de maladie respiratoire chronique avaient un séjour plus court avec une médiane de 8 jours et cette différence est statistiquement significative. $(\mathrm{P}=$ 0,001) test de Kruskall-Wallis(Tableau V).

\section{DISCUSSIONS}

Au cours de notre étude, la mortalité par rapport à l'ensemble des hospitalisations a été de $29,95 \%$. La tranche d'âge de 60-95 ans était la plus touchée $(32,3 \%)$. La prédominance masculine dans notre étude $(77,2 \%)$ avec un sex-ratio de 3,39. Parmi les patients qui avaient un antécédent médical connu, la tuberculose pulmonaire a été la plus représentée avec $32 \%$. Dans notre étude, les principaux diagnostics de décès étaient la tuberculose pulmonaire et les pneumopathies bactériennes avec respectivement $28,0 \%$ et $20,06 \%$. Dans notre étude, les lésions radiologiques étaient bilatérales dans 126 cas $(66,7 \%)$. Leurs 'étendues étaient supérieures au 2/3 du poumon dans 39,8\%. La durée du séjour moyen était de18, 6 jours avec des extrêmes de 3 et 183 jours et une médiane de 10 jours. La médiane de la durée de séjour chez les patients décédés par l'infection tuberculeuse a été de 15 jours et était plus longue que celle des maladies respiratoires chroniques avec une durée médiane de 8 jours et la différence était statistiquement significative $(\mathrm{p}=0,001)$. Dans notre étude, la mortalité par rapport à l'ensemble des hospitalisations a été de $29,95 \%$. Ce chiffre est nettement en dessous des $98,1 \%$ trouvés par thomas DELL'ACCIO à Grenoble [17] et au-dessus des $8,7 \%$ de Olufemi et al. au Nigéria [18], des 26,2\% de M. Ouédraogo et al. au Burkina Faso [19], et des $17 \%$ de N.O.Touré et al. à Dakar [20]. Ces décès peuvent s'expliquer par le retard avant la consultation, les références inadaptées, le manque d'équipement adéquat et l'absence d'éducation pour la santé. La tranche d'âge de 60-95 ans était la plus touchée $(32,3 \%)$, résultat comparable avec celui de Thomas DELL'ACCIO [17], à celui de N.O.Touré et al. [20] et à celui d'A. Diaw et coll. [21] en Côte d'ivoire. Par contre M. Ouédraogo et al. [20] de même que O. Olufemi et al. [19] ont trouvé une tranche d'âge de 25-44ans.La prédominance masculine dans notre étude $(77,2 \%)$ avec un sex-ratio de 3,39 a été également signalé par de nombreux auteurs: Thomas DELL'ACCIO [18], N.O.Touré et al. [20] et A. Diaw et al. [21]. Parmi les patients qui avaient un antécédent médical connu, la tuberculose pulmonaire a été la plus représentée avec $32 \%$ résultat non conforme à celui de A. Diaw qui a trouvé une prédominance des cancers [21]. Ces deux pays (Mali et Côte d'Ivoire) sont des zones d'endémie tuberculeuse mais la faiblesse du plateau technique et le faible revenu des patients s'expliqueraient le fait que les cancers soient encore sous-diagnostiqués au Mali. Dans notre étude, les principaux diagnostics de décès étaient la tuberculose pulmonaire et les pneumopathies bactériennes avec respectivement $28,0 \%$ et $20,06 \%$, contre $50,0 \%$ et $12,5 \%$ pour O.Olufemi et al. [18], par contre $33,8 \%$ et $44,9 \%$ ont été retrouvée par M. Ouédraogo et al. [19] avec les pneumopathies bactériennes en tête et pour DELL'ACCIO [17] le cancer broncho-pulmonaire arrivait en tête avec $66,66 \%$. Dans notre étude, les lésions radiologiques étaient bilatérales dans 126 cas $(66,7 \%)$.Leurs 'étendues étaient supérieures au $2 / 3$ du poumon dans $39,8 \%$. La durée du séjour moyen était de18, 6 jours avec des extrêmes de 3 et 183 jours et une médiane de 10 jours. Ces résultats sont comparables avec ceux de Thomas DELL'ACCIO [17] avec une moyenne de 13 jours et de O. Olufemi et al. [18] avec une moyenne de 14 jours. Elles sont supérieures à celle N.O.Touré et al. [20] avec une moyenne de 8 jours. Limites de l'étude : $\mathrm{Au}$ cours de notre étude, nous avons rencontré quelques contraintes, notamment une collecte difficile des données liée à leur mauvais archivage, parfois des examens para cliniques non faits ou égarés.

\section{CONCLUSION}

La mortalité chez les malades hospitalisés est élevée au service de Pneumo-phtisiologie du CHU $\mathrm{du}$ Point G. Le sexe masculin paye le plus lourd tribut surtout ceux du $3^{\mathrm{e}}$ âge. Les lésions radiologiques pulmonaires massives surtout bilatérales sont les facteurs les plus associées au décès. Le délai était moins de 10 jours dans plus de la moitié des cas. La tuberculose reste la cause la plus retrouvée.

\section{REFERENCES}

1- Agence régionale de la santé corse. Analyse de la morbi-mortalité en région corse. Version juin 2012: 23-25P.

2- Xian Sheng Chen et al. Analysis of basic data of the study on prevention and treatment of COPD.

Chinese Journal of tuberculosis respiratory disease, 1998, 21 (12): 749-752.

3- Organisation mondiale de la santé (OMS).Prévenir et combattre les maladies respiratoires aigües à tendance épidémique ou 
pandémique : Recommandation provisoire de l'OMS, juin 2007.

4- Nadia Aït-Khaled, Donald Enarson et Jean Bousquet. Les maladies respiratoires chroniques dans les pays en développement : charge de morbidité et stratégies de prévention et de prise en charge, Bulletin of the World Health Organisation, 2001, 79 (10) : 971-979.

5- La santé observée en Provence-Alpes-côte d'Azur. Les maladies respiratoires, fiche 7.10, Edition 1999.

6- ChristosChouaïd, Alain Vergnenègre. Données épidémiologiques des maladies respiratoires, Edition 2004.

7- HUCHON G. Enjeux de l'assistance respiratoires à domicile. Rev Prat 2001; 51 : 1105 1109.

8- Dr. Françoise LALANDE, Olivier VEBER. La mort à l'hôpital, Novembre 2009.

9- Aït-Khaled N et al. Epidemiology and management of stable chronic obstructive pulmonary disease in Africa In: Similowski T,

Derenne P. Clinical management of clinical obstructive pulmonary disease. New York, Marcel Dekker (sous presse).

10- Organisation Mondiale de la Santé (OMS). Plan mondial halte à la tuberculose 2011-2015 :

transformer la lutte vers l'élimination de la tuberculose, Edition 2011, 2p.

11- Horo K et al. Dynamique de la pathologie respiratoire dans le service de pneumologie en Afrique dans le contexte de l'infection VIH de 1998-2007 Revue pneumologique clinique 2011.
12- Ly BA. Morbidité et mortalité des services de médecine, de soins intensifs et de santé mentale. Thèse Med, Bamako, 2001 ; $\mathrm{N}^{\circ} 108.70 \mathrm{p}$. 13-Bassirou S. Profil de la morbidité et de la mortalité au centre de santé de référence Bougouni. Thèse Med, Bamako, 2006 ; 48p.

14- Organisation mondiale de la Santé. Maladies non transmissibles (MNT) : Profils de pays 2011. 15- Direction national de la santé. Système local d'information sanitaire : Profil du système de santé du Mali, février 2005.

16- Bagayoko Y. Motifs d'admission des patients au service de pneumo-phtisiologie du centre hospitalo-universitaire du point $\mathrm{G}$. Thèse Med, Bamako, 40-41P.

17- Thomas DELL'ACCIO. La revue de la morbidité et la mortalité dans un service de pneumologie et oncologie thoracique $\mathrm{CHU}$ Chambéry/sur une période de 4 ans. Thèse de Med. 2012, 55p

18- Olufemi D. et Coll. Respiratory diseases morbiolity and mortality among adults attending tertiary hospital in Nigeria. J. bras.pneumol.2009; $35: 8$

19- Ouédraogo M et Coll. Morbi-mortalité dans le service de pneumologie du CHU Yalgado Ouédraogo de Ouagadougou. Rév Mal. Respir $2009 ; 26: 88$

20- N.O. Touré et Coll. Mortalité dans un service de pneumologie de Dakar. Rév Mal Respir 2013 ; $30: 118$

21- A. DIAW et Coll. Profil des patients décédés en milieu pneumologique. Rév Mal Respir 2013 ; $30: 119$.

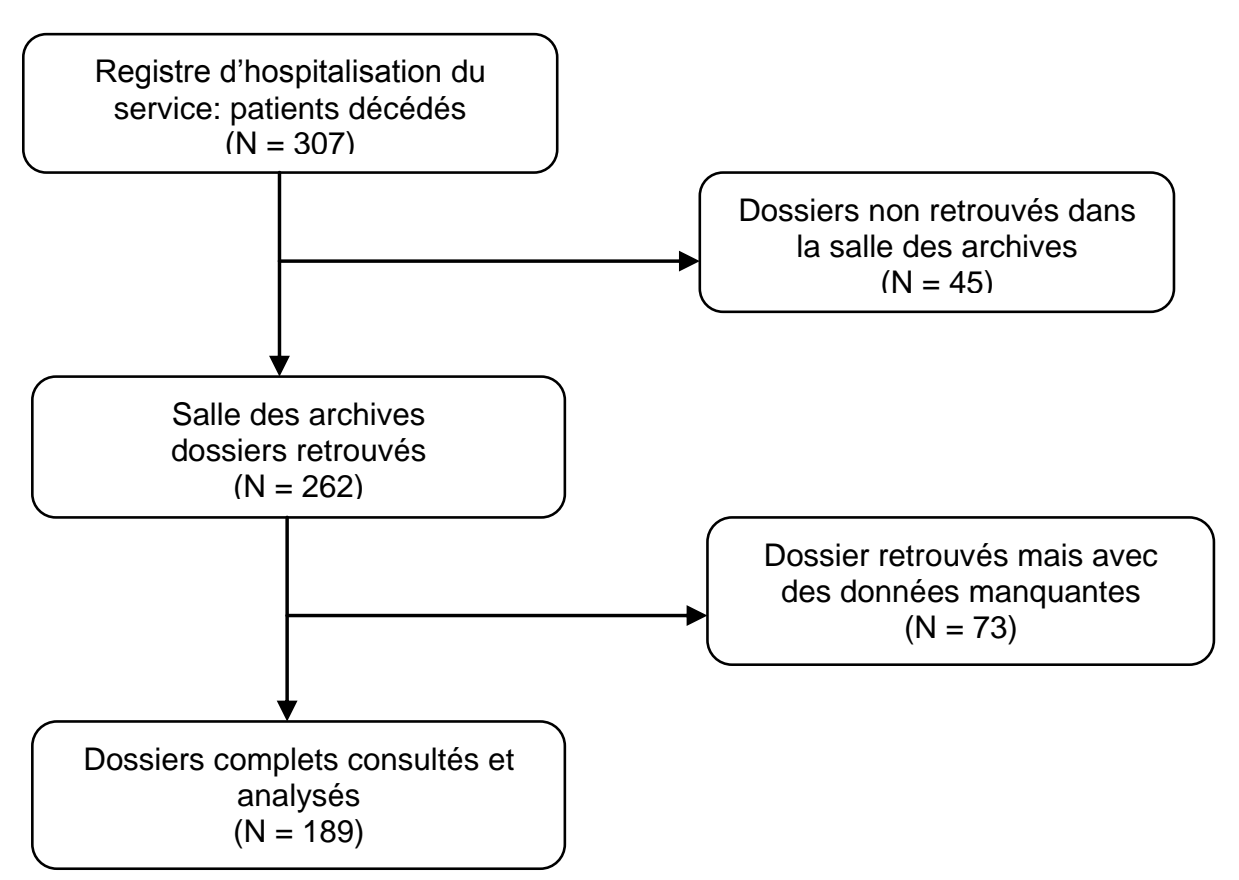

Figure1 : Etape de la collecte des dossiers 


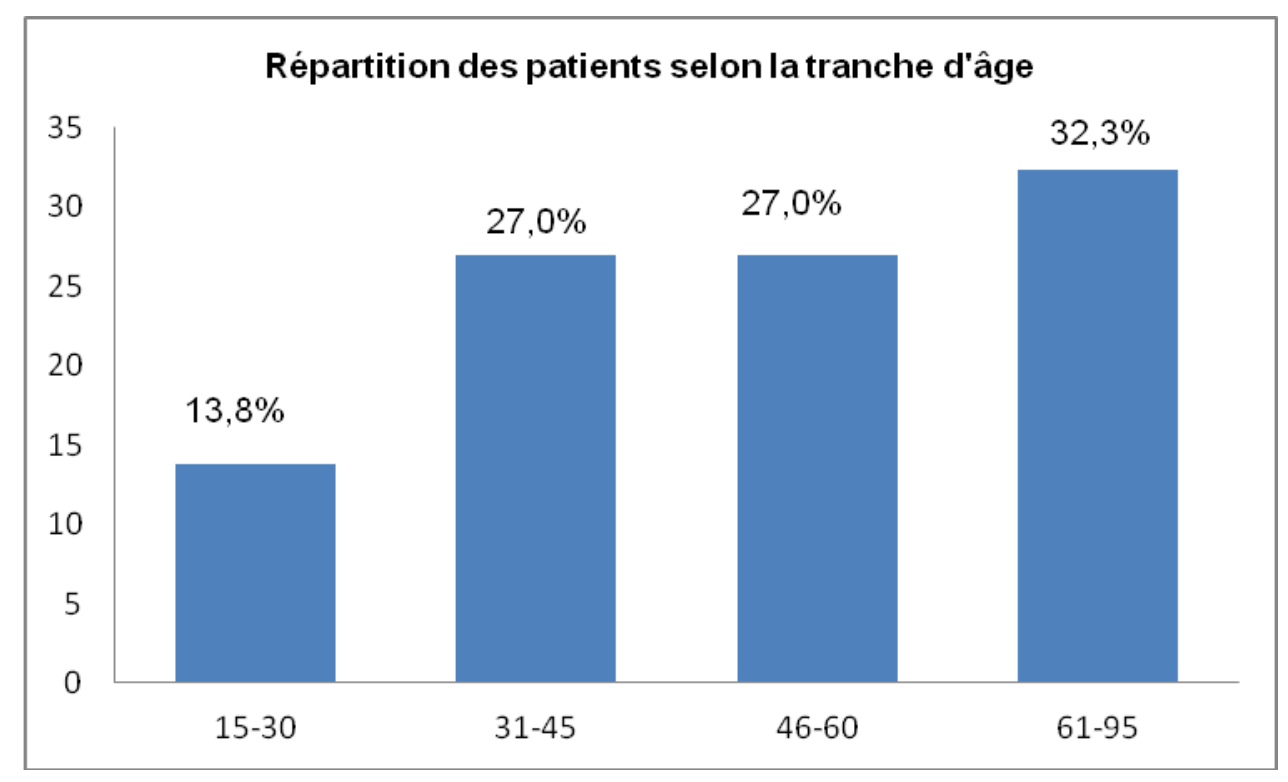

Figure 2- Répartition des patients selon la tranche d'âge

\section{Répartition en fonction du sexe}

Figure 3 : Répartition des patients selon le sexe 


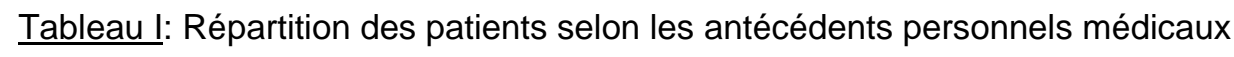

\begin{tabular}{lcc}
\hline ANTECEDENT PERSONNEL & $\begin{array}{c}\text { EFFECTIF } \\
\text { N=189 }\end{array}$ & POURCENTAGE \\
\hline Aucun & 117 & 61,9 \\
Tuberculose pulmonaire & 23 & 12,2 \\
Ulcère Gastroduodénal (UGD) & 22 & 11,6 \\
Hypertension artérielle (HTA) & 14 & 7,4 \\
Diabète & 4 & 2,1 \\
Asthme & 4 & 2,1 \\
$\begin{array}{l}\text { Broncho-pneumopathie chronique obstructive } \\
\text { (BPCO) } \\
\text { Drépanocytose }\end{array}$ & 3 & 1,6 \\
\hline
\end{tabular}

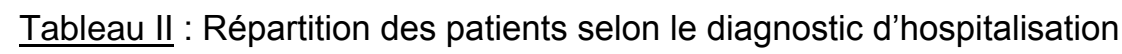

\begin{tabular}{lcc}
\hline \multicolumn{1}{c}{ DIAGNOSTIC D'HOSPITALISATION } & $\begin{array}{c}\text { EFFECTIF } \\
\mathrm{N}=189\end{array}$ & POURCENTAGE \\
\hline Pneumopathie bactérienne & 53 & 28,0 \\
Tuberculose pulmonaire & 47 & 24,9 \\
Pleurésie bactérienne & 30 & 15,9 \\
BPCO décompensée par une pleuro & 16 & 8,5 \\
pneumopathie bactérienne & 16 & 8,5 \\
Tuberculose extra pulmonaire & 11 & 5,8 \\
$\begin{array}{l}\text { Pneumopathie interstitielle fébrile et non } \\
\text { fébrile }\end{array}$ & 8 & 4,2 \\
Cancer broncho-pulmonaire & 4 & 2,1 \\
Hydro pneumothorax & 2 & 1,1 \\
Abcès pulmonaire & 2 & 1,1 \\
Asthme & 4 & \\
\hline
\end{tabular}


Tableau III : Répartition des patients selon l'étendue des lésions radiologiques

\begin{tabular}{lcc}
\hline ETENDUE DES LESIONS & $\begin{array}{c}\text { EFFECTIF } \\
\mathbf{N = 1 8 9}\end{array}$ & POURCENTAGE \\
\hline Supérieure à 2/3 & 74 & 39,8 \\
Entre $1 / 3-$ 2/3 & 65 & 34,9 \\
Inferieur ou égale à 1/3 & 47 & 25,3 \\
Absence des lésions & 3 & 1,6 \\
\hline
\end{tabular}

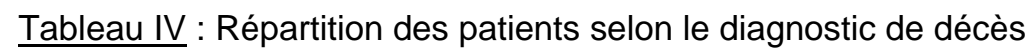

\begin{tabular}{lcc}
\hline DIAGNOSTIC DE DECES & $\begin{array}{c}\text { EFFECTIF } \\
\mathbf{N}=\mathbf{1 8 9}\end{array}$ & POURCENTAGE \\
\hline Tuberculose pulmonaire & 53 & 28,0 \\
Pneumopathie bactérienne & 39 & 20,6 \\
Pleurésie bactérienne & 23 & 12,2 \\
Tuberculose extra pulmonaire & 22 & 11,6 \\
Cancer broncho-pulmonaire & 18 & 9,5 \\
BPCO décompensée par une pleuro & 15 & 7,9 \\
pneumopathie bactérienne & 10 & 5,3 \\
Pneumopathie interstitielle & 5 & 2,6 \\
Hydro pneumothorax & 2 & 1,1 \\
Abcès pulmonaire & 1 & 0,5 \\
Asthme & 1 & 0,5 \\
*Autre & 189 & 100 \\
TOTAL & 5 & \\
\hline
\end{tabular}

${ }^{*}$ Autre (insuffisance cardiaque) 


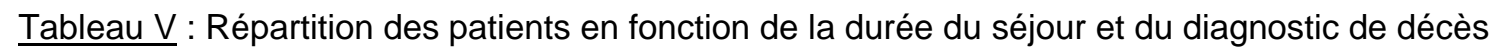

\begin{tabular}{lcccc}
\hline DIAGNOSTIC DE & EFFECTIF & DUREE & DEVIATION & MEDIANE \\
\hline DECES & $\mathrm{N}=189$ & MOYENNE & STANDARD &
\end{tabular}

Infection tuberculeuse

75

26,6800

33,6970

15

Pleuro-pneumopathie

79

14,1646

21,8012

9

bactérienne

Affection tumorale

19

13,5000

9,8054

9,5

Maladie respiratoire

16

9,3125

4,6842

8 chronique 